\title{
Fatores associados às necessidades de tratamento odontológico em idosos brasileiros: uma análise multinível
}

\author{
Factors associated with the need for oral treatment \\ of elderly Brazilians: a multilevel analysis
}

Chaiane Emilia Dalazen ${ }^{1}$

Alessandro Diogo De Carli ${ }^{1}$

Rafael Aiello Bomfim ${ }^{1}$

${ }^{1}$ Faculdade de Odontologia, Universidade Federal de Mato Grosso do Sul. Cidade Universitária s/n, Universitário. 79070-900 Campo Grande MS Brasil. chaiane_1@msn.com
Abstract The aims of this study were to estimate the prevalence of prosthetic requirements and dental treatment needs among the elderly Brazilian population and the associations with contextual and individual determinants. Data were taken from the Brazilian Oral Health Survey (SB Brasil 2010) and a sample of 7,619 Brazilian people aged 65-74 years were investigated. Multilevel logistic regression models were used to estimate the odds ratio (OR) and 95\% confidence intervals between treatment needs and contextual variables (Municipal Human Development Index, Gini coefficient and oral health coverage in the Family Health Strategy) and individual (gender, income, education and skin color). The prevalence of treatment need ranged from municipalities ( $p<$ 0.05). Of the individual variables, gender was associated with dental treatment need $(O R=1.18$, 95\% CI 1.05 to 1.31); lower education and nonwhite skin were risk factors $(O R=1.18 ; 95 \% C I$ 1.05 to 1.31 and $O R=1.28,95 \%$ CI 1.15 to 1.43 ); the non-white skin color represented a risk factor $(O R=1.83 ; 95 \% C I 1,38-2,42)$ and for the need for prosthesis income was a protection factor $(O R$ $=0.39 ; 95 \%$ CI 0, 30-.51). The results showed an association between the individual factors and treatment needs.

Key words Socioeconomic factors, Dental health surveys, Family health program, Health service coverage
Resumo Este estudo teve como objetivos estimar a prevalência da necessidade de prótese e tratamento dental em idosos brasileiros, e verificar a associação entre essas necessidades e fatores contextuais e individuais. Realizou-se estudo com dados $(n=7.619)$ da Pesquisa Nacional de Saúde Bucal - SB Brasil 2010. Modelos de regressão logística multinível foram utilizados para estimar odds ratio e intervalos de $95 \%$ de confiança entre as necessidades de tratamento e as variáveis contextuais (Índice de Desenvolvimento Humano Municipal, Coeficiente de Gini e cobertura de saúde bucal na Estratégia de Saúde da Família) $e$ individuais (sexo, renda, escolaridade e cor da pele). Para a necessidade de tratamento dental, ser homem, apresentar menor escolaridade, menor renda, cor da pele autorrelatada como não branca e residir em municípios com menor IDH - $M$ foram fatores que aumentaram as chances de os indivíduos necessitarem de tratamento; para a necessidade de prótese, a cor da pele autorreferida como não branca, menor renda e menor escolaridade também aumentaram as chances dos idosos necessitarem de prótese. Os resultados indicaram associação entre as necessidades de tratamento $e$ determinantes individuais e contextuais.

Palavras-chave Fatores socioeconômicos, Saúde bucal, Idoso, Inquéritos de saúde bucal, Estratégia de saúde da família, Cobertura de serviços públicos de saúde 


\section{Introdução}

O envelhecimento populacional é um fenômeno mundial ${ }^{1,2}$ e, no Brasil, nas últimas três décadas, o número de pessoas idosas cresceu, em média, nove vezes ${ }^{3}$. Esta transição demográfica demanda mudanças na organização do sistema de saúde e é um desafio aos gestores e profissionais da saúde pública $^{4}$. Nesse sentido, é relevante investigar as condições de saúde bucal dos idosos, pois, para parcela significativa desta população, ainda há dificuldade de acesso à atenção odontológica, associada a problemas de saúde bucal decorrentes da exposição à práticas mutiladoras ao longo da vida e à percepção de que perdas dentárias são inerentes ao processo de envelhecimento $0^{5,6}$.

O processo saúde-doença é permeado por questões complexas vinculadas ao contexto, modo de vida e características individuais das populações, sujeitas às desigualdades, que, no campo da saúde são dinâmicas e refletem o impacto de múltiplos determinantes ${ }^{7}$. A distribuição das desigualdades sociais e iniquidades em saúde devem ser consideradas ao planejar medidas de prevenção, monitoramento e tratamento dos agravos bucais ${ }^{8}$. O interesse por pesquisas sobre a relação entre iniquidades e saúde bucal não é recente e aumentou a partir das evidências de que as doenças bucais são mais frequentes em grupos populacionais em desvantagem social ${ }^{9}$.

Nesta perspectiva, os levantamentos epidemiológicos são ferramentas de grande importância para a vigilância em saúde, sendo fundamentais para a construção de bancos de dados que indiquem os principais agravos em saúde ${ }^{10}$. Em 2010, o Ministério da Saúde realizou o quarto levantamento epidemiológico em saúde bucal, denominado SB Brasil 2010, que avaliou uma amostra representativa da população brasileira; estudos com a abrangência do SB Brasil podem auxiliar a compreensão dos problemas de saúde bucal e disponibilizar informações para subsidiar a formulação de políticas públicas ${ }^{11}$.

O objetivo deste estudo foi de estimar a prevalência da necessidade de prótese e tratamento dental em idosos brasileiros, e verificar a associação entre estas necessidades e determinantes contextuais e individuais.

\section{Materiais e Métodos}

Trata-se de estudo quantitativo, analítico, de corte transversal, baseado em dados secundários oriundos do levantamento epidemiológico SB
Brasil 2010 e de características contextuais das capitais, Distrito Federal e dos municípios do interior avaliados no referido levantamento. Foram utilizados dados referentes às necessidades de tratamento odontológico de idosos, com idade de 65 a 74 anos $(n=7.619)$.

O SB Brasil 2010 utilizou técnica de amostragem probabilística por conglomerado, em que cada capital das unidades da federação e o Distrito Federal foram consideradas um domínio $(\mathrm{n}=27)$ e todos os municípios do interior, de cada região, outro domínio $(n=5)$. Cada domínio do interior foi composto por 30 municípios $^{11}$. Foram realizados exames odontológicos com luz natural, espelho bucal plano e sonda periodontal. As equipes de campo que realizaram os exames $\mathrm{e}$ entrevistas foram compostas por um cirurgiãodentista e um anotador, treinados e calibrados previamente e com concordância entre os examinadores calculada, sendo o coeficiente Kappa mínimo aceitável com valor de $0,65^{11}$.

No presente estudo, as variáveis dependentes analisadas foram a necessidade de tratamento dental e a necessidade de prótese ${ }^{11}$. Para a análise bivariada e multinível, em relação à necessidade de tratamento dental, foram excluídos os idosos edêntulos $(\mathrm{n}=3.583)$ e os idosos sem informação $(\mathrm{n}=110)$. Para a análise bivariada e multinível da necessidade de prótese, foram excluídos os idosos sem informação $(n=3.642)$.

A necessidade de tratamento dental foi categorizada em: "necessita ou não necessita", e para sua determinação foram verificadas as questões do banco de dados do SB Brasil 2010 (NT1, NT2, NT3, NT5, NT6). Estas correspondem, respectivamente, à necessidade de restauração de uma superfície dentária, restauração de duas ou mais superfícies dentárias, necessidade de prótese unitária, necessidade de tratamento pulpar e restauração e necessidade de extração. Foram excluídos os idosos edêntulos desta análise. A necessidade de prótese foi categorizada em: "não necessita e necessita", a partir da necessidade de prótese total e parcial removível, obtidas através da questão do banco de dados (NECPROT) ${ }^{11}$.

As características contextuais escolhidas para analisar as desigualdades sociais incluíram o Índice de Desenvolvimento Humano Municipal (IDHM) e o Coeficiente de Gini, referentes ao ano de $2010^{12}$. A cobertura populacional estimada pelas equipes básicas de saúde bucal na Estratégia de Saúde de Família (SB/ESF), no ano de 2010, foi utilizada como um indicador de oferta e facilidade de acesso de serviços de odontologia básica ${ }^{13}$. A análise também considerou a variável domínio 
que fez referência ao município no qual o idoso residia, se era capital ou município do interior. Todos estes dados são de domínio público, relativos às capitais, DF e aos 150 municípios do interior avaliados pelo SB Brasil 2010 11 . A cobertura pelas equipes SB/ESF foi categorizada em: menor que $25 \%$, entre $25 \%$ e $50 \%$, e maior que $50 \%$.

O IDHM é uma medida geométrica das dimensões de renda, educação e longevidade, cujo valor varia de 0 a 1: quanto maior o valor, melhores são as condições sociais. O Coeficiente de Gini é uma medida estatística de desigualdade de distribuição de renda que varia entre 0 e 1 , em que 0 corresponde à igualdade absoluta e 1 à desigualdade absoluta ${ }^{12}$. O IDHM foi categorizado em: de 0,5 - 0,7 e maior que 0,7; e Coeficiente Gini categorizado em: menor que 0,5 e de 0,5 - 0,7

Os determinantes individuais investigados foram sexo, cor da pele autorreferida, escolaridade e renda familiar ${ }^{11}$. A escolaridade foi avaliada através do número de anos de estudos completos sem reprovação e categorizada em: até quatro anos de estudo e cinco anos ou mais. A cor da pele autorreferida classificada em branca e não branca (preta, parda e outros). A renda familiar foi categorizada em: até R\$1.500,00 e superior a R\$ $1.500,00$.

Inicialmente, foram estimadas as prevalências das necessidades de tratamento que, posteriormente, foram analisadas através de testes quiquadrado de Pearson. Para a obtenção da distribuição espacial das necessidades de tratamento dos idosos no Brasil, dados das prevalências de necessidade de tratamento dos municípios (capitais e interior), de cada unidade federativa, foram submetidos ao software de geoprocessamento QGIS $^{\circledast}$ (Creative Commons Attribution-Share) versão 2.1, juntamente com os índices de malhas digitais dos municípios brasileiros do Instituto Brasileiro de Geografia e Estatística (IBGE) ${ }^{14}$.

A associação entre as variáveis dependentes e independentes foi analisada através de modelos de regressão logística multinível realizadas no software Stata ${ }^{\circledR}$, versão 13.0 (StataCorp., CollegeStation, Estados Unidos), considerando o plano complexo de amostragem e pesos amostrais. A correção pelo efeito de desenho foi feita utilizando o comando Svy do Stata ${ }^{\varpi}$ para análise de amostras complexas, que constou de domínios relativos às capitais e municípios do interior, totalizando 32 domínios. As unidades da amostra foram estratificadas em 2 estratos, sendo as do nível superior correspondentes aos 32 domínios, aos referidos pesos e população finita de correção e as do nível inferior correspondentes aos valores de necessidade de tratamento e necessidade de prótese dos indivíduos.

A regressão logística multinível utilizou modelos de efeitos mistos (aleatórios e fixos) com intercepto aleatório e com função logit (mixed effects) para obtenção das medidas das razões de chances (odds ratio - OR) e intervalo de confiança de 95\% (IC95\%). Inicialmente, foram estimadas associações bivariadas entre as variáveis contextuais e individuais e as necessidades de tratamento. Após as associações bivariadas, o critério para a manutenção das variáveis nos modelos multiníveis finais foi o valor de $\mathrm{p}<0,05$.

O Projeto SB Brasil 2010 foi conduzido dentro dos padrões exigidos pela Declaração de Helsinque e aprovado pelo Conselho Nacional de Ética em Pesquisa, em janeiro de 2010. Esta pesquisa foi submetida à análise do Comitê de Ética em Pesquisa (CEP) da Universidade Federal de Mato Grosso do Sul (UFMS) e aprovada.

\section{Resultados}

A amostra total de idosos do projeto SB Brasil 2010 foi de 7.619 indivíduos, sendo que destes, $61,9 \%$ eram do sexo feminino, $47,2 \%$ autorrelataram cor da pele branca e 58\% haviam completado até o $4^{\mathrm{a}}$ ano do ensino fundamental. A renda familiar declarada de maior frequência foi de até R\$ 1.500,00 (66,4\%). Em relação aos determinantes contextuais dos municípios, 43,2\% desta população residiam em municípios com cobertura de equipes de saúde bucal na ESF entre 25\% e 50\%. Quanto ao Coeficiente de Gini dos municípios, 91,3\% dos idosos residiam em municípios com alto Coeficiente de Gini $(0,5-0,7)$, e $88,3 \%$ em municípios com IDHM alto $(>0,7)$, conforme Tabela 1. Entre os avaliados, 49\% não possuíam nenhum dente natural e 48\% necessitavam de prótese total e/ou parcial. Entre os não edêntulos, 59,5\% possuíam necessidade de tratamento dental, conforme a Tabela 2 .

$O$ teste do qui-quadrado de Pearson, com nível de significância de 5\%, identificou diferença estatisticamente significativa entre as prevalências de necessidades de tratamento dental de cada domínio analisado $(\mathrm{p}<0,05)$. Para a população em questão, a prevalência das necessidades de tratamento dental, entre as capitais, variou de 43\%, em Porto Alegre e Goiânia, a 81\%, em Belém. Nos municípios do interior, a menor prevalência foi observada na região Sul (54\%), enquanto a maior foi nas regiões Nordeste e Centro -Oeste (72\%), conforme Figura 1. 
Tabela 1. Análise descritiva das variáveis contextuais, individuais e necessidades de tratamento em idosos, Brasil, 2010.

\begin{tabular}{|c|c|}
\hline Variáveis & n (\%) \\
\hline \multicolumn{2}{|l|}{ Contextuais } \\
\hline \multicolumn{2}{|l|}{ Coeficiente de Gini } \\
\hline$<0,5$ & $663(8,7)$ \\
\hline $0,5-0,7$ & $6.956(91,3)$ \\
\hline \multicolumn{2}{|l|}{ IDHM } \\
\hline $0,5-0,7$ & $891(11,7)$ \\
\hline$>0,7$ & $6.728(88,3)$ \\
\hline \multicolumn{2}{|l|}{ Cobertura SB/ESF } \\
\hline$<25 \%$ & $3.002(39,4)$ \\
\hline $25 \%-50 \%$ & $3.291(43,2)$ \\
\hline$>50 \%$ & $1.326(17,4)$ \\
\hline \multicolumn{2}{|l|}{ Domínio } \\
\hline Capital & $5.973(78,4)$ \\
\hline Interior & $1.646(21,6)$ \\
\hline \multicolumn{2}{|l|}{ Individuais } \\
\hline \multicolumn{2}{|l|}{ Sexo } \\
\hline Masculino & $2.903(38,1)$ \\
\hline Feminino & $4.716(61,9)$ \\
\hline \multicolumn{2}{|c|}{ Cor da pele autorreferida } \\
\hline Branca & $3.596(47,2)$ \\
\hline Não branca & $4.023(52,8)$ \\
\hline \multicolumn{2}{|l|}{ Renda familiar } \\
\hline$<\mathrm{R} \$ 1.500,00$ & $5.059(66,4)$ \\
\hline$>\mathrm{R} \$ 1.500,00$ & $2.560(33,6)$ \\
\hline \multicolumn{2}{|c|}{ Escolaridade (anos de estudo) } \\
\hline$\geq 5$ anos & $3.200(42,0)$ \\
\hline $0-4$ anos & $4.419(58,0)$ \\
\hline \multicolumn{2}{|c|}{ Necessidades de tratamento } \\
\hline \multicolumn{2}{|c|}{ Dental } \\
\hline Sim & $2.337(30,7)$ \\
\hline Não & $5.172(67,9)$ \\
\hline Sem informação & $110(1,4)$ \\
\hline \multicolumn{2}{|l|}{ Prótese } \\
\hline Sim & $3.622(48,1)$ \\
\hline Não & $303(4,1)$ \\
\hline Sem informação & $3.642(47,8)$ \\
\hline
\end{tabular}

Para a necessidade de prótese, houve diferença estatisticamente significativa entre os municípios ( $\mathrm{p}<0,01$, teste do qui-quadrado de Pearson). A prevalência de necessidade de prótese variou entre os municípios do interior de 56\% no interior da região Norte a $38 \%$ no interior da região Sul. Em relação às capitais, a maior prevalência foi verificada em Belém (71\%) e a menor em Goiânia (31\%), conforme Figura 2.

$\mathrm{Na}$ análise não ajustada entre a necessidade de tratamento dental e as variáveis contextuais
Tabela 2. Análise descritiva das variáveis contextuais, individuais e necessidades de tratamento em idosos não edêntulos $(\mathrm{n}=3.926)$, Brasil, 2010

\begin{tabular}{|c|c|}
\hline Variáveis & n (\%) \\
\hline \multicolumn{2}{|l|}{ Contextuais } \\
\hline \multicolumn{2}{|l|}{ Coeficiente de Gini } \\
\hline$<0,5$ & $254(6,5)$ \\
\hline $0,5-0,7$ & $3.672(93,5)$ \\
\hline \multicolumn{2}{|l|}{ IDHM } \\
\hline $0,5-0,7$ & $351(9)$ \\
\hline$>0,7$ & $3.575(91)$ \\
\hline \multicolumn{2}{|l|}{ Cobertura SB/ESF } \\
\hline$<25 \%$ & $1.666(42,5)$ \\
\hline $25 \%-50 \%$ & $1.693(43,1)$ \\
\hline$>50 \%$ & $567(14,4)$ \\
\hline \multicolumn{2}{|l|}{ Domínio } \\
\hline Capital & $3.264(83,1)$ \\
\hline Interior & $662(16,9)$ \\
\hline \multicolumn{2}{|l|}{ Individuais } \\
\hline \multicolumn{2}{|l|}{ Sexo } \\
\hline Masculino & $1.645(41,9)$ \\
\hline Feminino & $2.281(58,1)$ \\
\hline \multicolumn{2}{|c|}{ Cor da pele autorreferida } \\
\hline Branca & $1.897(48,3)$ \\
\hline Não branca & $2.029(51,7)$ \\
\hline \multicolumn{2}{|l|}{ Renda familiar } \\
\hline$<\mathrm{R} \$ 1.500,00$ & $2.397(61)$ \\
\hline$>\mathrm{R} \$ 1.500,00$ & $1.529(39)$ \\
\hline \multicolumn{2}{|c|}{ Escolaridade (anos de estudo) } \\
\hline$\geq 5$ anos & $1.942(49,5)$ \\
\hline $0-4$ anos & $1.984(50,5)$ \\
\hline \multicolumn{2}{|c|}{ Necessidades de tratamento } \\
\hline \multicolumn{2}{|c|}{ Dental } \\
\hline Sim & $2.337(59,5)$ \\
\hline Não & $1.589(40,5)$ \\
\hline \multicolumn{2}{|l|}{ Prótese } \\
\hline Sim & $2.478(63,1)$ \\
\hline Não & $285(7,3)$ \\
\hline Sem informação & $1.163(29,6)$ \\
\hline
\end{tabular}

e individuais, todas as variáveis apresentaram significância estatística $(\mathrm{p}<0,05)$, com exceção do Coeficiente de Gini, conforme Tabela 3. No modelo final da análise multinível, modelo ajustado, as variáveis IDHM, sexo, cor da pele autorreferida, renda e escolaridade permaneceram estatisticamente significativas após ajuste pelas demais variáveis. Mulheres tiveram chance 33\% menor de necessitar de tratamento $(\mathrm{OR}=0,0,67$; IC95\% 0,58 - 0,77). Idosos que autorreferiram a cor da pele como não branca (OR = 1,46; IC95\% 


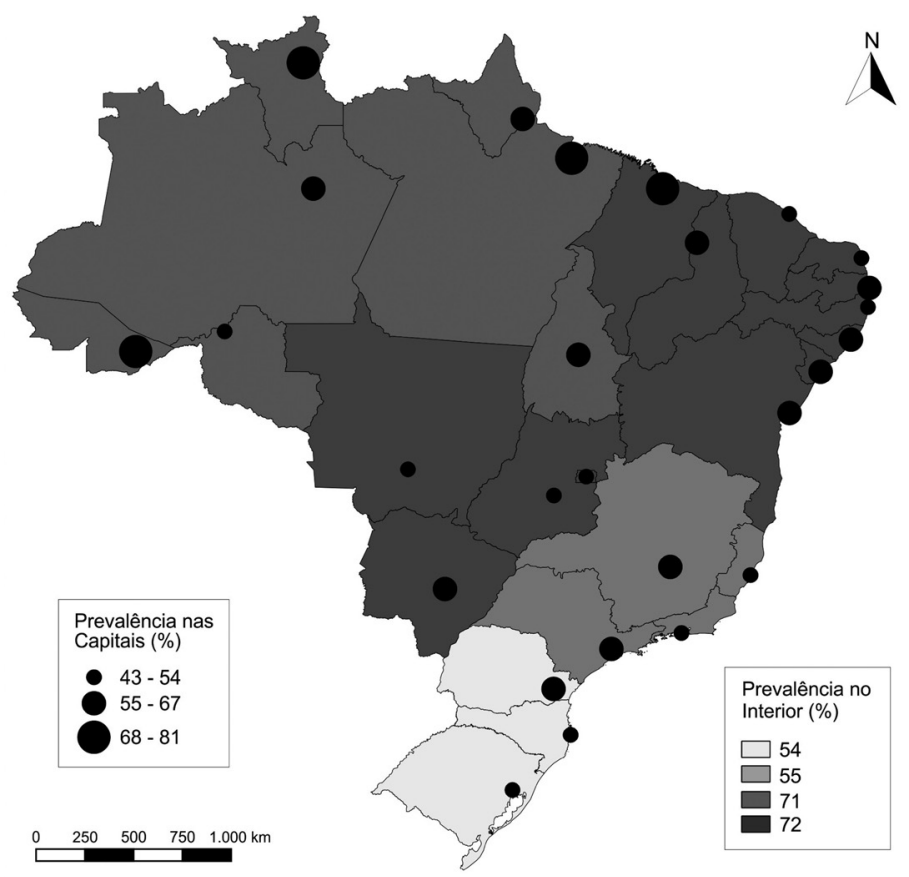

Figura 1. Distribuição espacial da prevalência de necessidade de tratamento dental em idosos brasileiros. SB Brasil, 2010.

1,26 - 1,69) apresentavam menor escolaridade $(\mathrm{OR}=1,69 ; \mathrm{IC} 95 \% 1,60-1,70)$ e menor renda $(\mathrm{OR}=0,64 ;$ IC95\% 0,55- 0,74) apresentaram maiores chances de necessitar de tratamento, conforme Tabela 3.

Em relação à necessidade de prótese, na análise não ajustada, as variáveis IDHM, domínio, cor da pele, renda e escolaridade apresentaram significância estatística $(\mathrm{p}<0,10)$, sendo incluídas no modelo ajustado, conforme Tabela 4. Após ajuste das variáveis contextuais e individuais entre si, a análise da associação entre determinantes contextuais e individuais e a necessidade de prótese revelou que as variáveis contextuais não apresentaram associação estatisticamente significativa. Assim, apresentaram chances maiores de necessitar de prótese os idosos que autorrelatam cor da pele como não branca $(\mathrm{OR}=2.22$; IC95\% 1.65 $-2,97)$ e com menor escolaridade $(\mathrm{OR}=1,30$; IC95\% 1,22-1,41). Aqueles que relataram maior renda tiveram chance $67 \%$ menor de necessitar de prótese $(\mathrm{OR}=0,33$; IC95\% 0,30-0,51), conforme Tabela 4.

\section{Discussão}

Apesar da ampliação do acesso aos serviços odontológicos, promovida pela Política Nacional de Saúde Bucal (Brasil Sorridente), a prevalência das necessidades de tratamento dental (59,5\%) e de prótese (48\%) de parcela de idosos da população brasileira ainda é elevada.

Neste estudo, consideramos as iniquidades em saúde como diferenças entre grupos populacionais, as quais resultam de condições externas e não podem ser controladas pelos indivíduos, sendo consideradas sistemáticas, evitáveis, injustas e desnecessárias ${ }^{15}$. Nesse aspecto, os resultados encontrados evidenciaram que os sujeitos que residiam em municípios com menor IDHM, do sexo masculino, que autorreferiram a cor da pele como não-branca e com menor escolaridade apresentaram chances maiores de necessitar de tratamento, indicando a existência de iniquidades em saúde bucal entre os idosos brasileiros, corroborando com os resultados de pesquisas anteriores em diferentes países, que também constataram a influência de fatores socioeconômicos nas condições de saúde bucal da população ${ }^{16,17}$. 


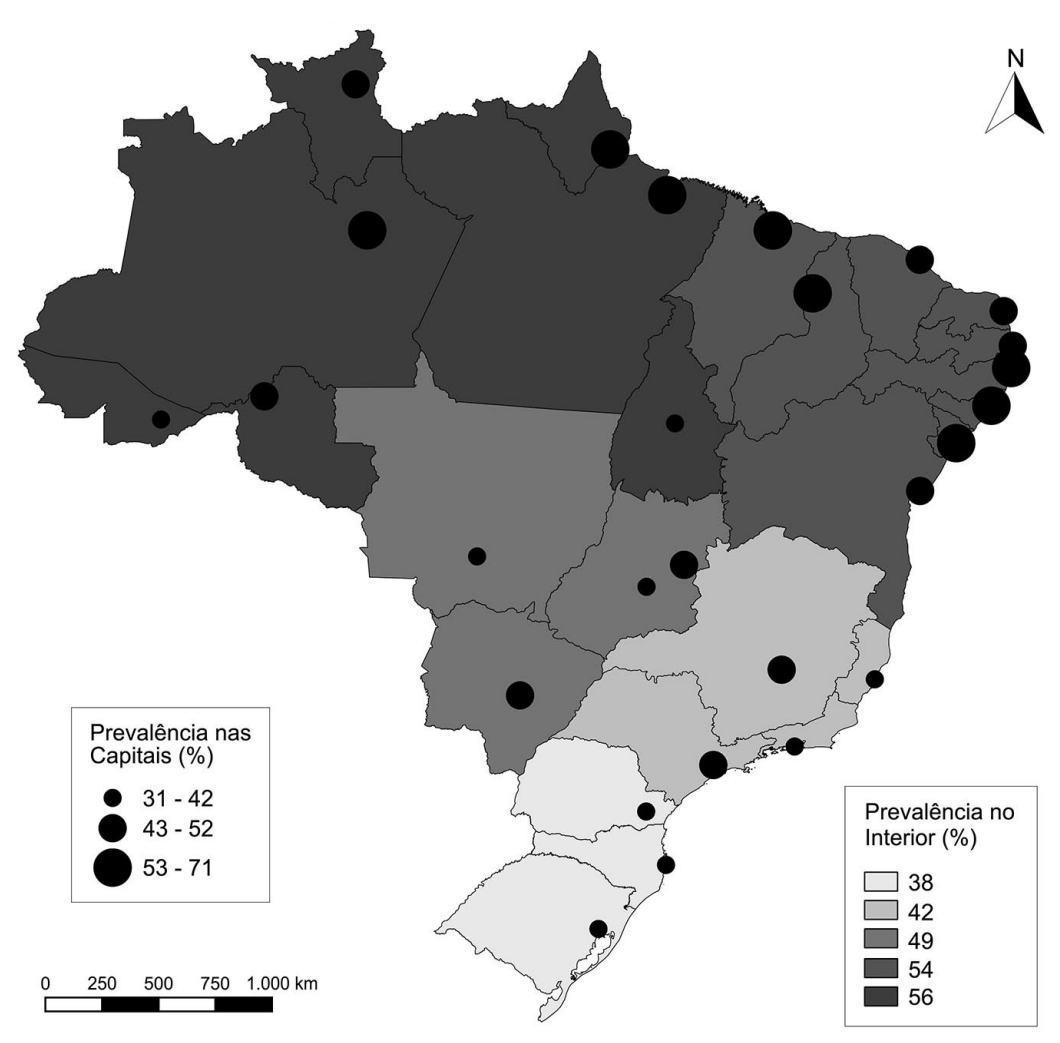

Figura 2. Distribuição espacial da prevalência de necessidade de prótese em idosos brasileiros. SB Brasil, 2010.

Nesta análise, a escolaridade esteve associada à necessidade de tratamento dental e de prótese. O tempo de estudo é um aspecto que influencia o acesso e a utilização dos serviços de saúde, tendo em vista que indivíduos com maior escolaridade apresentam maior acesso aos serviços de saúde, procurando os mesmos com maior frequência, e, além disso, o total de anos de estudo também está associado à adoção de atitudes mais saudáveis $^{18,19}$. Entre os idosos que participaram do SB Brasil 2010, foram identificados baixos níveis de escolaridade, sendo que $58 \%$ deles tinham até quatro anos de estudo, fato que pode influenciar o perfil precário de saúde bucal desta população. A questão de gênero também pode estar relacionada a estes resultados, pois mais de $60 \%$ destes idosos eram do sexo feminino, e os mesmos ainda carregam as marcas de uma cultura que não priorizou o acesso à educação formal entre as mulheres, com variações nos níveis de escolaridade marcadas por diferenças de gênero ${ }^{20}$.

Iniquidades também foram identificadas nas variáveis sexo e cor da pele autorrelatada, sendo que homens e não brancos apresentaram maio- res necessidades de tratamento. Estes achados são uníssonos aos resultados de estudos anteriores $^{21-23}$, corroborando com o fato de que mulheres podem apresentar menor necessidade de tratamento porque se preocupam mais com sua saúde bucal e procuram atendimento odontológico com maior frequência ${ }^{18,22,24}$. Nessa perspectiva, também pode existir a influência de questões de gênero em relação à saúde bucal de idosos, como as diferenças entre os papéis de homens e mulheres, presentes no imaginário social, que atribuem o cuidado e a prevenção como próprios do sexo feminino, cabendo, aos homens, as dificuldades de acesso aos serviços de saúde, devido ao horário de funcionamento e às cobranças do mercado de trabalho ${ }^{25}$.

As maiores necessidades de tratamento dental e de prótese entre os participantes do levantamento, que autorrelataram a cor da pele como não branca, refletem iniquidades em sua dimensão social, às quais alguns grupos étnicos estão submetidos no Brasil ${ }^{25}$. Estudos indicam que as piores condições de saúde bucal podem ser resultado da ação de fatores que ainda afetam negros e 
Tabela 3. Análise bivariada e modelo final da regressão logística multinível dos determinantes da necessidade de tratamento dental em idosos, SB Brasil, 2010

\begin{tabular}{|c|c|c|c|c|c|c|}
\hline \multirow{2}{*}{ Variáveis } & \multicolumn{3}{|c|}{ Análise bivariada } & \multicolumn{3}{|c|}{ Modelo final da regressão multinível } \\
\hline & OR & IC95\% & $\mathbf{p}$ & OR & IC95\% & p \\
\hline \multicolumn{7}{|l|}{ Contextuais } \\
\hline \multicolumn{7}{|l|}{ Coeficiente de Gini } \\
\hline$<0,5$ & 1 & & & - & - & - \\
\hline $0,5-0,7$ & 1,00 & $0,76-1,3$ & 0,97 & - & - & - \\
\hline \multicolumn{7}{|l|}{ IDHM } \\
\hline $0,5-0,7$ & 1 & & & 1 & & \\
\hline$>0,7$ & 0,3 & $0,41-0,68$ & 0,000 & 0,56 & $0,37-0,86$ & 0,008 \\
\hline \multicolumn{7}{|l|}{ Cobertura SB/ESF } \\
\hline$<25 \%$ & 1 & & & 1 & & \\
\hline $25 \%-50 \%$ & 0,98 & $0,85-1,13$ & 0,86 & 0,99 & $0,69-1,40$ & 0,99 \\
\hline$>50 \%$ & 1,2 & $1,00-1,55$ & 0,03 & 0.83 & $0,57-1,20$ & 0,33 \\
\hline \multicolumn{7}{|l|}{ Domínio } \\
\hline capital & 1 & & & 1 & & \\
\hline interior & 1,0 & $1,09-1,56$ & 0,002 & 0,84 & $0,55-1,28$ & 0,42 \\
\hline \multicolumn{7}{|l|}{ Individuais } \\
\hline \multicolumn{7}{|l|}{ Sexo } \\
\hline Masculino & 1 & & & 1 & & \\
\hline Feminino & 0,66 & $0,58-0,76$ & 0,000 & 0,67 & $0,58-0,77$ & 0,000 \\
\hline \multicolumn{7}{|l|}{ Cor da pele } \\
\hline Branca & 1 & & & 1 & & \\
\hline Não branca & 1,6 & $1,63-2,12$ & 0,000 & 1,46 & $1,26-1,69$ & 0,000 \\
\hline \multicolumn{7}{|l|}{ Renda familiar } \\
\hline$\leq \mathrm{R} \$ 1.500$ & 1 & & & 1 & & \\
\hline$>\mathrm{R} \$ 1.500$ & 0,5 & $0,44-0,58$ & 0,000 & 0,64 & $0,55-0,74$ & 0,000 \\
\hline \multicolumn{7}{|l|}{ Escolaridade } \\
\hline$\geq 5$ anos & 1 & & & 1 & & \\
\hline $0-4$ anos & 0,5 & $0,48-0,62$ & 0,000 & 1,69 & $1,60-1,70$ & 0,000 \\
\hline
\end{tabular}

* Domínio var 0.1 (IC95\%0.05-0.2). Log likeli: -2510.4961.

outras etnias, como menores escolaridade, renda e acesso aos serviços de saúde ${ }^{26,27}$.

Entre os municípios avaliados pelo SB Brasil 2010, a prevalência das necessidades de tratamento dental em idosos variou de $43 \%$ a $81 \%$. As maiores prevalências foram encontradas em capitais da região Norte e Nordeste, que apresentavam baixas coberturas populacionais de SB/ ESF, menores do que 50\%. As marcantes desigualdades regionais identificadas pelo presente estudo podem estar relacionadas à baixa cobertura populacional pelas equipes de saúde bucal na ESF. Porém, não se pode deixar de considerar os efeitos das dimensões continentais do Brasil, que dificultam a administração e resultam em amplas desigualdades socioeconômicas regionais ${ }^{28,29}$. A região Nordeste é a região mais pobre do país, seguida pela região Norte ${ }^{28}$ e, apesar de haver evidências de que as diferenças regionais têm diminuído nas últimas décadas ${ }^{29}$, seus efeitos ainda podem estar presentes na prevalência das necessidades de tratamento odontológico entre os idosos brasileiros. Estes, provavelmente, ainda refletem em termos de processo saúde-doença, o modo e as condições de vida de toda uma geração que vivenciou uma situação econômica, política e paradigmática, em relação à Odontologia, que desfavorecia a manutenção da saúde bucal.

A prevalência de edentulismo dos idosos avaliados pelo SB Brasil 2010, de 47\%, está entre as mais elevadas do mundo ${ }^{8}$. Não houve redução significativa na prevalência de perdas dentárias dos idosos brasileiros avaliados pelo SB Brasil 2010, em relação aos idosos avaliados pelo le- 
Tabela 4. Análise bivariada e modelo final da regressão logística multinível dos determinantes da necessidade de prótese em idosos $(n=3.926)$, Brasil, 2010

\begin{tabular}{|c|c|c|c|c|c|c|}
\hline \multirow{2}{*}{ Variáveis } & \multicolumn{3}{|c|}{ Análise bivariada } & \multicolumn{3}{|c|}{ Modelo final da regressão multinível } \\
\hline & OR & IC95\% & p & OR & IC95\% & $\mathrm{p}$ \\
\hline \multicolumn{7}{|l|}{ Contextuais } \\
\hline \multicolumn{7}{|l|}{ Coeficiente de Gini } \\
\hline$<0,5$ & 1 & & & - & - & - \\
\hline $0,5-0,7$ & 0,64 & $0,35-1,07$ & 0,08 & - & - & - \\
\hline \multicolumn{7}{|l|}{ IDHM } \\
\hline $0,5-0,7$ & 1 & & & 1 & & \\
\hline$>0,7$ & 0,36 & $0,19-0,61$ & 0,001 & 1,34 & $0,54-3,36$ & 0,521 \\
\hline \multicolumn{7}{|l|}{ Cobertura SB/ESF } \\
\hline$<25 \%$ & 1 & & & & & \\
\hline $25 \%-50 \%$ & 0,86 & $0,62-1,19$ & 0,36 & - & - & - \\
\hline$>50 \%$ & 1,2 & $1,00-1,55$ & 0,01 & - & - & - \\
\hline \multicolumn{7}{|l|}{ Domínio } \\
\hline capital & 1 & & & 1 & & \\
\hline interior & 2,13 & $1,47-3,12$ & 0,000 & 1,64 & $0,67-3,98$ & 0,273 \\
\hline \multicolumn{7}{|l|}{ Individuais } \\
\hline \multicolumn{7}{|l|}{ Sexo } \\
\hline Masculino & 1 & & & - & - & - \\
\hline Feminino & 1 & $0,79-1,28$ & 0,93 & - & - & - \\
\hline \multicolumn{7}{|l|}{ Cor da pele } \\
\hline Branca & 1 & & & 1 & & \\
\hline Não branca & 3,91 & $3,00-5,13$ & 0,000 & 2,22 & $1,65-2,97$ & 0,000 \\
\hline \multicolumn{7}{|l|}{ Renda familiar } \\
\hline$\leq \mathrm{R} \$ 1.500$ & 1 & & & 1 & & \\
\hline$>\mathrm{R} \$ 1.500$ & 0,18 & $0,14-0,23$ & 0,000 & 0,33 & $0,25-0,44$ & 0,000 \\
\hline \multicolumn{7}{|l|}{ Escolaridade } \\
\hline$\geq 5$ anos & 1 & & & 1 & & \\
\hline $0-4$ anos & 0,17 & $0,13-0,23$ & 0,000 & 1,30 & $1,22-1,41$ & 0,000 \\
\hline
\end{tabular}

* Domínio var 0.38(IC95\% 0.16-0.86). Log likeli: -878.79.

vantamento de 2003 (SB Brasil 2003) ${ }^{8}$. De acordo com a Política Nacional de Saúde da Pessoa Idosa, o SUS ainda está distante de dar respostas efetivas e eficazes às necessidades e demandas de saúde da pessoa idosa ${ }^{30}$, sendo que a análise do acúmulo de doenças bucais não tratadas pode propiciar aos formuladores de políticas públicas informações necessárias para uma melhor reflexão sobre as necessidades da população ${ }^{18}$.

Entretanto, em relação à necessidade de prótese, o poder de inferência dessa variável foi comprometido no presente estudo, devido à falta de informações no banco de dados do SB Brasil 2010 (não há informação sobre a necessidade de prótese de cerca de $47 \%$ dos idosos avaliados). A ausência de informação sinaliza para uma fragilidade do SB 2010, que deve ser superada nas pró- ximas edições, sendo importante a identificação das causas desse problema, desde a calibração dos examinadores até o processo de coleta e armazenamento dos dados. Apesar do baixo poder de inferência, foi identificada necessidade de prótese em $48 \%$ dos participantes do levantamento, podendo ser considerada herança dos modelos assistenciais centrados em práticas curativas e mutiladoras, responsável pelo grande número de idosos edêntulos e, consequentemente, pela alta demanda por serviços especializados ${ }^{31}$.

De acordo com Peres et al. ${ }^{22}$, a partir dos anos de 1980 e 1990, houve uma melhoria nas condições socioeconômicas da população e no sistema de saúde, o que promoveu uma redução das perdas dentárias, principalmente de crianças e adolescentes. Entretanto, os idosos avaliados 
no último levantamento epidemiológico nacional, SB Brasil $2010^{11}$, não viveram essas melhorias durante a infância e adolescência e, por isso, estima-se que uma redução do edentulismo nos idosos seja significativa, somente, a partir do ano de 2050.

Em relação à necessidade de prótese, também foram identificadas iniquidades regionais. As maiores prevalências de necessidade de prótese foram identificadas nos municípios das regiões Norte e Nordeste. O Sistema Único de Saúde (SUS) tem apresentado dificuldades em suprir a demanda por serviços especializados, mesmo com a ampliação do número de Centros de Especialidades Odontológicas (CEOs) e de Laboratórios de Prótese. Até o ano de 2010, havia aproximadamente 853 CEOs e 664 unidades de laboratórios de prótese dentária ${ }^{32}$. Em 2013, dos 890 CEOs existentes no Brasil, 352 estavam na região Nordeste e apenas 53 na região $\mathrm{Norte}^{33}$. A implantação dos CEOs deveria apoiar-se na realidade epidemiológica de cada região, procurando atingir maiores coberturas nos grupos populacionais que mais necessitam de sua intervenção $0^{34}$.

Levando em consideração a distribuição geográfica, a melhor relação habitante/CEO é a da região Nordeste, sendo, em 2010, a Paraíba o estado brasileiro com maior cobertura - 71,1 mil habitantes/CEO, enquanto a média nacional de cobertura de CEO é de cerca de 200,5 mil habitantes para cada $\mathrm{CEO}^{35}$. É possível que o princípio da equidade tenha norteado a distribuição dos CEOs no Nordeste, devido às elevadas necessidades de tratamento da população e às desigualdades sociais, mas o mesmo não foi aplicado à região Norte ${ }^{33}$. Outra hipótese presente na literatura é a de que a Região Nordeste foi contemplada com elevado número de CEOs por ser pioneira na implantação de políticas públicas de saúde no Brasil ${ }^{36}$.

Apesar de ser a região com maior número de CEOs do Brasil, os municípios do Nordeste incluídos no SB Brasil 2010 apresentaram prevalências de necessidade de prótese $\geq 50 \%$. Neste aspecto, outros fatores devem ser considerados como a cobertura populacional pela Estratégia de Saúde da Família e a interface entre a atenção primária e a atenção secundária dos serviços odontoló$\operatorname{gicos}^{32}$. Barreiras de acesso na atenção primária podem dificultar também o acesso aos serviços especializados. A maior cobertura da atenção básica no território em que o serviço especializado está implantado é um dos fatores relevantes para a garantia da integralidade na assistência à saúde bucal nos $\mathrm{CEOs}^{37}$. Entraves no processo de refe- rência entre a atenção básica e outros níveis de complexidade também podem interferir na redução das necessidades da população ${ }^{33}$.

Após o ajuste para características individuais, a associação entre o IDHM e as necessidades de tratamento se manteve. Idosos residentes em municípios com piores condições socioeconômicas apresentaram maiores chances de necessitar de tratamento. A associação entre iniquidades sociais e os desfechos em saúde bucal já foram identificadas em estudos anteriores e estão relacionadas a maiores dificuldades de acesso aos serviços ${ }^{38,39}$. Tal fato é relevante, pois, para os idosos, principalmente aqueles com piores condições financeiras, qualquer dificuldade no acesso aos serviços de saúde pode se tornar um obstáculo capaz de impedir ou interromper a continuidade do cuidado em saúde ${ }^{39}$.

Na última década, houve significativo aumento da incorporação do atendimento odontológico no SUS, principalmente através da inclusão das equipes de saúde bucal na ESF, o que deveria possibilitar a adoção de uma postura mais ativa, não limitando o serviço público odontológico ao atendimento clínico, mas incluindo a atuação na comunidade, principalmente, com ações preventivas e de educação em saúde ${ }^{40}$. Apesar disso, há poucos dados de amplitude nacional que permitam analisar a existência de algum efeito dessa ampliação da cobertura do serviço público odontológico sobre as necessidades de tratamento dos idosos. Diante dessa realidade, a cobertura pelas equipes de saúde bucal na ESF foi incluída como uma variável do presente estudo. Não foi identificada associação entre a cobertura populacional de ESB/ESF e as necessidades de tratamento em idosos, indicando que apenas a ampliação da cobertura não é suficiente para a redução das necessidades em saúde bucal dos idosos.

Os achados referentes à cobertura populacional, pelas equipes de saúde bucal na ESF, sugerem, respeitando-se as limitações de um estudo transversal, o fato de que, em função das mudanças no perfil demográfico populacional brasileiro, o SUS, aqui representado pela ESF, tem, como relevante desafio, o de prover atenção à saúde bucal da população dessa faixa etária, de modo a otimizar questões relacionadas ao processo saúde-doença bucal, o que é viável por ações de promoção e educação em saúde, alicerces da ESF e da $\mathrm{PNSB}^{40}$, e também por práticas assistenciais voltadas para suprir as demandas preventivas e curativas/reabilitadoras dessa faixa etária.

Apesar de fornecer informações importantes sobre as necessidades de tratamento odontológi- 
co dos idosos, não se pode deixar de considerar as limitações deste estudo. Devido ao desenho do estudo, transversal, os resultados obtidos referenciam hipóteses relativas aos fatores associados, mas não têm poder de inferência causal. Assim como, embora o SB Brasil 2010 tenha abrangência nacional e seja o maior banco de dados disponível sobre as condições de saúde bucal da população brasileira, as análises provenientes de seus resultados não estão isentas de limitações, como a falta de informação sobre a necessidade de prótese de quase metade dos idosos brasileiros avaliados.

Os resultados desta pesquisa demonstraram alta prevalência de necessidades de tratamento odontológico em idosos brasileiros e indicaram associações entre as necessidades de tratamento e fatores socioeconômicos e demográficos, sendo que a influência destes determinantes variou de acordo com a necessidade de saúde bucal avaliada. Essas informações podem contribuir para o processo de tomada de decisão e possibilitar um melhor planejamento da alocação de recursos, com possível direcionamento de recursos adicionais para as áreas que manifestam necessidades mais elevadas ${ }^{21,41}$.

Políticas públicas são delineadas em um modo de construção social a partir de interesses em disputa que geram conflitos e debates entre grupos e sujeitos. Desta forma, pode-se considerar que as políticas de saúde não estão distantes do contexto social em que são formuladas ${ }^{41}$. A saúde bucal dos brasileiros é reflexo de um contexto histórico, que por muito tempo não priorizou a prestação de serviços odontológicos públicos ${ }^{21}$, inclusive os referentes à população idosa.

\section{Conclusões}

A prevalência da necessidade de prótese entre os idosos variou de $31 \%$ a $71 \%$ e a prevalência da necessidade de tratamento dental de $43 \%$ a $81 \%$. Identificou-se variação significativa da prevalência das necessidades de tratamento e de prótese entre as cidades brasileiras. A necessidade de prótese apresentou, por regressão logística multinível, associação significativa à cor da pele autorreferida, renda e escolaridade, enquanto a necessidade de tratamento dental esteve associada ao sexo, renda, escolaridade, cor da pele autorreferida e ao IDHM. Sugere-se que a ampliação da cobertura da ESF seja estrategicamente planejada para contemplar as necessidades em saúde bucal dos idosos brasileiros.

\section{Colaboradores}

CE Dalazen foi responsável pela coleta dos dados secundários e redação do texto. AD De Carli foi responsável pelo desenvolvimento da pesquisa e redação e revisão do texto e o autor RA Bomfim foi responsável pela análise estatística e revisão do texto. 


\section{Referências}

1. Gülcan F, Ekback G, Ordell S, Lie SA, Åstrom AN. Inequality in oral health related to early and later life social conditions: a study of elderly in Norway and Sweden. BMC Oral Health 2015; 15:20.

2. Hernández-Palacios RD, Ramírez-Amador V, Jarillo-Soto EC, Irigoyen-Camacho ME, Mendoza-Núñez VM. Relationship between gender, income and education and self-perceived oral health among elderly Mexicans. An exploratory study. Cien Saude Colet 2015; 20(4):997-1004

3. Beltrão KI, Camarano AA, Kanso S. Dinâmica populacional brasileira na virada do século XX. Rio de Janeiro: IPEA; 2004.

4. Veras R. Envelhecimento populacional contemporâneo: demandas, desafios e inovações. Rev Saude Publica 2009; 43(3):548-554

5. Silva SRC, Fernandes RAC. Autopercepção das condições de saúde bucal por idosos. Rev Saude Publica 2001; 35:349-55.

6. Moreira RS, Nico LS, Tomita NE, Ruiz T. A saúde bucal do idoso brasileiro: revisão sistemática sobre o quadro epidemiológico e acesso aos serviços de saúde bucal. Cad Saude Publica 2005; 21(6):1665-1675.

7. Sadana R, Blas E. What can public health programs do to improve health equity? Public Health Rep 2013; 128(Supl. 3):12-20.

8. Peres MA, Barbato PR, Reis SCGB, De Morais Freitas CHS, Antunes JLF. Perdas dentárias no Brasil: análise da Pesquisa Nacional de Saúde Bucal 2010. Rev Saude Publica 2013; 47(3):78-89.

9. Locker D. Deprivation and oral health: a review. Community Dent Oral Epidemiol 2000; 28(3):161-169.

10. Roncalli AG, Côrtes MIS, Peres KG. Perfis epidemiológicos de saúde bucal no Brasil e os modelos de vigilância. Cad Saude Publica 2012; 28(Supl.):S58-S68.

11. Brasil. Ministério da Saúde (MS). Secretaria de Atenção à Saúde. Departamento de Atenção Básica. Coordenação Nacional de Saúde Bucal. SB Brasil 2010: Pesquisa Nacional de Saúde Bucal: Resultados principais, 2011 [acessado 2013 Dez 3]. Disponível em: http://bvsms. saude.gov.br/bvs/publicacoes/pesquisa_nacional_saude_bucal.pdf

12. Programa das Naç̃os Unidas para o Desenvolvimento (PNUD). Atlas de desenvolvimento humano no Brasil: 1991-2010. 2013. [acessado 2013 Dez 20]. Disponível em: http://www.pnud.org.br/IDH/Atlas2013.aspx?indiceAccordion=1\&li=li_Atlas2013

13. Brasil. Ministério da Saúde (MS). Datasus. Informações de Saúde. Cobertura equipes de Saúde bucal, 2010. [acessado 2014 Jan 10]. Disponível em: http://tabnet. datasus.gov.br/cgi/dh.exe?pacto/cnv/pactbr.def

14. Instituto Brasileiro de Geografia e Estatística. Malhas digitais dos municípios brasileiros, 2013. [acessado 2014 Jan 10]. Disponível em: ftp://geoftp.ibge.gov.br/ malhas_digitais/municipio_2013/

15. Whitehead M. The concepts and principles of equity and health. Geneva: WHO, 2000.

16. Steele J, Shen J, Tsakos G, Fuller E, Morris S, Watt R, Guarnizo-Herreño C, Wildman J. The Interplay between Socioeconomic Inequalities and Clinical Oral Health. J Dent Res 2015; 94(1):19-26.
17. Fonseca FA, Jones KM, Mendes DC, Dos Dantos Neto PE, Ferreira RC, Pordeus IA, Martins AMBL. The oral health of seniors in Brazil: addressing the consequences of a historic lack of public health dentistry in an unequal society. Gerodontology 2013; 31(1):1-10.

18. Marmot M, Bell R. Social Determinants and Dental Health. Adv Dent Res 2011; 23(2):201-206.

19. Parahyba MI, Veras R, Melzer D. Incapacidade funcional entre as mulheres idosas no Brasil. Rev Saude Publica 2005; 39(3):383-391.

20. Barbato PR, Nagano HCM, Zanchet FN, Boing AF, Peres MA. Perdas dentárias e fatores sociais, demográficos e de serviços associados em adultos brasileiros: uma análise dos dados do Estudo Epidemiológico Nacional (Projeto SB Brasil 2002-2003). Cad Saude Publica 2007; 23(8):1803-1814.

21. Vettore MV, Marques RAA, Peres MA. Desigualdades sociais e doença periodontal no estudo SB Brasil 2010: abordagem multinível. Rev Saude Publica 2013; 47(3):29-39.

22. Peres KG, Cascaes AM, Leão ATT, Cortes MIS, Vettore MV. Aspectos sociodemográficos e clínicos da qualidade de vida relacionada à saúde bucal em adolescentes. Rev Saude Publica 2013; 47(Supl. 3):19-28.

23. Gomes R, Nascimento EF, Araujo FC. Por que os homens buscam menos os serviços de saúde do que as mulheres? As explicações de homens com baixa escolaridade e homens com ensino superior. Cad Saude Publica 2007; 23(3):565-574.

24. Peres MA, Antunes JLF, Boing AF, Peres KG, Bastos JLD Skin colour is associated with periodontal disease in Brazilian adults: a population-based oral health survey. J Clin Periodontol 2007; 34(3):196-201.

25. Souza EHA, Oliveira PAP, Paegle AC, Goes PSA. Raça e o uso dos serviços de saúde bucal por idosos. Cien Saude Colet 2012; 17(8):2063-2070.

26. Paim JS, Travassos CMR, Almeida CM, Bahia L, Macinko J. The Brazilian health system: history, advances, and challenges. Lancet 2011;377(9779):1778-1797.

27. Victora C, Barreto ML, Leal MC, Monteiro CA, Schmidt MI, Paim J, Bastos FI, Almeida C, Bahia L, Travassos C, Reichenheim M, Barros FC; Lancet Brazil Series Working Group. Health conditions and health-policy innovations in Brazil: the way forward. Lancet 2011; 377(9782):2042-2053

28. Brasil. Portaria no 2528 , de 18 de outubro de 2006. Política Nacional de Saúde da Pessoa Idosa. Diário Oficial da União 2006; 19 out.

29. Matos DL, Lima-Costa MF. Auto-avaliação da saúde bucal entre adultos e idosos residentes na Região Sudeste: resultados do Projeto SB-Brasil, 2003. Cad Saude Publica 2006; 22(8):1699-1707.

30. Saliba NA, Nayme JGR, Moimaz SAS, Cecilio LPP, Garbin CAS. Organização da demanda de um centro de especialidades odontológicas. Rev Odontol UNESP 2013; 42(5):317-323.

31. Machado FCA, Silva JV, Ferreira MAF. Fatores relacionados ao desempenho de Centros de Especialidades Odontológicas. Cien Saude Colet 2015; 20(4):11491163. 
32. Chaves SCL, Barros SG, Cruz DN, Figueiredo ACL, Moura BLA, Cangussu MCT. Política Nacional de Saúde Bucal: Fatores associados à integralidade do cuidado. Rev Saude Publica 2010; 44(6):1005-1013.

33. Lynch J, Smith GD, Harper S, Hillemeier M, Ross N, Kaplan GA, Wolfson M. Is Income Inequality a Determinant of Population Health? Part 1. A Systematic Review. Milbank Q 2004; 82(1):5-99.

34. Martins AB, D'avila OP, Hilgert JB, Hugo FN. Atenção Primária a Saúde voltada as necessidades dos idosos: da teoria à prática. Cien Saude Colet 2014; 19(8):34033416.

35. Martelli PJL, Cabral APS, Pimentel FC, MCLSV, Monteiro IS, Silva SF. Análise do modelo de atenção à saúde bucal em municípios do estado de Pernambuco. Cien Saude Colet 2008; 13(5):1669-1674.

36. Saliba NA, Moimaz SAS, Fadel CB, Bino LS. Saúde Bucal no Brasil: uma nova política de enfrentamento para a realidade nacional. ROBRAC 2010; 18(48):62-66.

37. Antunes JLF, Narvai PC. Políticas de saúde bucal no Brasil e seu impacto sobre as desigualdades em saúde. Rev Saude Publica 2010; 44(2):360-365.

38. Zermiani TC, Pimentel BV, Buffon MDCM, Ditterich RG. Indicadores de desenvolvimento humano e de saúde bucal na atenção básica nos municípios da região metropolitana de Curitiba-PR. Revista da Faculdade de Odontologia-UPF 2014; 19(2):185-192.

39. Fischer TK, Peres KG, Kupek E, Peres MA. Indicadores de atenção básica em saúde bucal. Rev. bras. epidemiol. 2010; 13(1):126-138.

40. Brasil. Ministério da Saúde (MS). Diretrizes da Política Nacional de Saúde Bucal. Brasília: MS; 2004.

41. Andrade LM, Sena ELS, Pinheiro GML, Meira EC, Lira LSSP. Políticas públicas para pessoas idosas no Brasil: uma revisão integrativa. Cien Saude Colet 2013; 18(12):3543-3552.

Artigo apresentado em 28/10/2015

Aprovado em 20/06/2016

Versão final apresentada em 22/06/2016 